\title{
RANK 1 ASPHERICAL MANIFOLDS WHICH DO NOT SUPPORT ANY NONPOSITIVELY CURVED METRIC
}

\author{
C. S. ARAVINDA AND F. T. FARRELL
}

\section{INTRODUCTION}

The celebrated theorem of Preissmann says that if $M$ is a compact manifold carrying a riemannian metric of strictly negative curvature then the fundamental group $\pi_{1}(M)$ of $M$ has the property $(\mathrm{P})$ that every non-trivial abelian subgroup is isomorphic to $\mathbb{Z}$. Since $M$ is also a $K\left(\pi_{1}(M), 1\right.$ )-manifold (cf. [Mi, p.103]) it is determined up to homotopy by $\pi_{1}(M)$. More generally, it was proved in [FJ1, Corollary 10.5] that, in dimensions bigger than $4, \pi_{1}(M)$ determines $M$ up to homeomorphism type. Hence one would expect strong inter-relations between the algebraic structure of $\pi_{1}(M)$ and the geometry of $M$, when $M$ is a closed $K\left(\pi_{1}(M), 1\right)$-manifold, and a natural question (cf. [BK, (6.2.1)]) to ask would be the following: If $\pi_{1}(M)$ has property $(\mathrm{P})$, does $M$ necessarily admit a metric of negative curvature? While this question still remains open, we prove, in this paper, that there are a large class of closed manifolds $M$ which are $K\left(\pi_{1}(M), 1\right)$-manifolds and such that $\pi_{1}(M)$ shares all the properties of the fundamental group of certain compact nonpositively curved manifolds whereas $M$ does not admit any metric of nonpositive curvature. In fact, we construct two classes of examples of compact topological manifolds $M$ supporting at least two distinct smooth structures $\mathcal{M}_{1}$ and $\mathcal{M}_{2}$ where $\mathcal{M}_{1}$ is a compact, rank 1 (in the sense of [BBE]) nonpositively curved manifold while $\mathcal{M}_{2}$ cannot support a nonpositively curved metric. These constructions are motivated by and closely follow the paper [FJ4] where exotic smoothings of complete, finite volume, real hyperbolic manifolds which do

Research supported in part by the National Science Foundation 
not support any complete, finite volume, pinched negatively metrics were constructed.

In $\S 2$, we discuss the first of these examples. We begin by recalling a certain construction of E. Heintze (cf. [BBE, p. 174]) of compact, rank 1, nonpositively curved $m$-dimensional manifolds $M$ which contain isometrically immersed flat $(m-1)$-tori. Then, following [FJ3], we try to change the differential structure on $M$ by removing an embedded tube $S^{1} \times \mathbb{D}^{m-1}$ from $M$ and then reinserting it with a 'twist' on the boundary of the tube. That is, let $f: S^{1} \times \mathbb{D}^{m-1} \rightarrow M$ be a smooth embedding and $\phi: S^{m-2} \rightarrow S^{m-2}$ be an orientation-preserving diffeomorphism. The new smooth manifold $M_{f, \phi}$ is obtained as a quotient space of the disjoint union

$$
S^{1} \times \mathbb{D}^{m-1} \coprod M-f\left(S^{1} \times \operatorname{Int} \mathbb{D}^{m-1}\right)
$$

where we identify points $(x, v)$ and $f(x, \phi(v))$ if $(x, v) \in S^{1} \times S^{m-2}$. Here, Int $\mathbb{D}^{m-1}$ denotes the interior of $\mathbb{D}^{m-1}$ and $\partial \mathbb{D}^{m-1}$ is identified with $S^{m-2}$. The smooth manifold $M_{f, \phi}$ is canonically homeomorphic to $M$ but is not always diffeomorphic to $M$. In fact, the Flat torus theorem can be used to show that some of these smooth manifolds $M_{f, \phi}$ do not support a nonpositively curved metric.

$\S 3$ deals with the second class of examples constructed by changing the smooth structure on certain of V. Schroeder's construction [S] of compact, rank 1 , nonpositively curved $m$-manifolds $M(m \geq 5)$ which contain totally geodesically embedded flat tori of codimension 2 . Here, we follow a topological approach to change the differential structure. We use the fact that there is a one- one correspondence (cf. [FJ2]) between concordance classes of smooth structures on $M$ and the homotopy classes of maps from $M$ to TOP/0 (denoted by $[M, T O P / 0])$, provided $m \geq 5$, with the original Schroeder smooth structure on $M$ corresponding to the class of the constant map. Changing the smooth structure on $M$ by constructing a suitable map from $M$ to $T O P / 0$, we again invoke the Flat torus theorem to argue out that this smooth structure on $M$ cannot support any nonpositively curved metric.

Acknowledgements: This research was done while the second author was a visiting faculty member at the Tata Institute of Fundamental Research. He 
wishes to thank the School of Mathematics, TIFR for its kind invitation and hospitality which made this collaboration possible.

\section{EXAmples From Heintze's CONSTRUCtion}

Consider a complete, finite volume, non-compact, connected, constant negatively curved $m$-dimensional Riemannian manifold. For simplicity, assume it has only one cusp. The cross-section of the cusp is a compact, flat $(m-1)$ dimensional submanifold $T$. Cut off the cusp and flatten the manifold near the cut to make it locally isometric to the direct product of $T$ and the unit interval. Now consider another copy of this and glue the two together along $T$. The resulting manifold has nonpositive sectional curvature and is compact containing an isometrically immersed flat $(m-1)$-torus inside it. For our purposes here we begin with a real hyperbolic finite volume $m$-manifold having at least two cusps and follow the above procedure at each cusp seperately to get a compact manifold $M$ with sectional curvature $\leq 0$. Considered only as a smooth manifold, $M$ is the double of the given hyperbolic manifold with its cusps cut off. Since the portion where the two copies were glued together is important for our discussions we refer to it as neck. Now pick a smooth closed path in $M$, with trivial normal bundle, passing through one of the necks where the two copies were glued together such that the path intersects the cross section $T$ of the neck transversally in exactly one point. Note that one can always find such a path passing through any designated $T$. Take a tubular neighborhood of this path and let $f: S^{1} \times \mathbb{D}^{m-1} \rightarrow M$ denote the embedding of this tubular neighborhood in $M$. We can now state,

Theorem 2.1. Let $M, T$ and $f: S^{1} \times \mathbb{D}^{m-1} \rightarrow M$ be as above and $m \geq 7$. If the diffeomorphism $\phi: S^{m-2} \rightarrow S^{m-2}$ represents a non trivial element of $\Theta_{m-1}$, the group of oriented diffeomorphism classes of oriented homotopy $(m-1)$ spheres and $T$ is a torus, then the smooth manifold $M_{f, \phi}$ is

1. homeomorphic to $M$, and

2. does not support any metric of nonpositive sectional curvature.

Before setting off to prove the theorem we recall and prove a few preliminary lemmas necessary for both the proof of the theorem and to show that its 
hypotheses are frequently satisfied. Corollary 2.4 will also be used in $\S 3$.

Lemma 2.2. Let $X$ be a complete, finite volume real hyperbolic manifold with one cusp $C$. Then there exists a finite sheeted covering space $X^{\prime}$, i.e., $\pi: X^{\prime} \rightarrow$ $X$, of $X$ corresponding to a finite index normal subgroup $K$ of $\pi_{1}(X)$ such that $X^{\prime}$ is a finite volume real hyperbolic manifold having more than one cusp.

Proof. The cusp $C$ topologically has the structure $T \times[0, \infty)$ where $T$ is a compact, flat, codimension 1 submanifold of $X$. Denote by $\Gamma$ and $S$ the fundamental groups of $X$ and $T$ respectively. It is known that $S$ is a subgroup of $\Gamma$. If $\phi: \Gamma \rightarrow G$ is the projection map onto the finite quotient group $G(=\Gamma / K)$, then we claim that the covering space $X^{\prime}$ has more than one cusp provided $\phi(S) \neq G$. This is easily verified by restricting the covering map $\pi$ to the closed subspace $\pi^{-1}(C)$ of $X$ and observing that $\pi^{-1}(C)$ is connected if and only if $S=\pi_{1}(C)$ acts transitively on the fibers. Furthermore, the number of connected components of $\pi^{-1}(C)$ is precisely equal to the cardinality of the coset space $G / \phi(S)$.

We next show that it is possible to find such coverings that, with the notation as above, $\phi(S) \neq G$. Now, $S$ being the fundamental group of a compact, flat, $(n-1)$ - dimensional manifold, is an extension of a finite group $F$ by $\mathbb{Z}^{n-1}$. That is, we have the following short exact sequence

$$
1 \rightarrow \mathbb{Z}^{n-1} \rightarrow S \rightarrow F \rightarrow 1 .
$$

Let $k$ be the order of the finite group $F$. Since $\Gamma$ contains a nonabelian free subgroup (cf. [BE, Theorem A]), there exist elements $a, b$ which freely generate a nonabelian free group $F_{2}$ contained in $\Gamma$. Put $g=\left[a^{k}, b^{k}\right]$. Since $g \neq 1$, by residual finiteness of $\Gamma$, (cf. [Ma, p. 309]), there exists a finite group $G$ and an epimorphism $\phi: \Gamma \rightarrow G$ such that $\phi(g) \neq 1$. Now, if $\phi(S)=G$, then there exist $x, y \in S$ with $\phi(x)=\phi(a)$ and $\phi(y)=\phi(b)$. Consequently, both $\phi\left(a^{k}\right), \phi\left(b^{k}\right) \in \phi\left(\mathbb{Z}^{n-1}\right)$ which is abelian. Therefore, $1=\left[\phi\left(a^{k}\right), \phi\left(b^{k}\right)\right]=\phi(g)$ which is a contradiction. Therefore we must have $\phi(S) \neq G$. Thus, the finite covering space $X^{\prime}$ of $X$ corresponding to the finite index subgroup $K=\operatorname{ker}(\phi)$ of $\Gamma$ has more than one cusp. 
Lemma 2.3. Let $S$ be a Bieberbach group of rank $n-1$, i.e., the fundamental group of a closed, flat $(n-1)$-dimensional manifold and $\phi: S \rightarrow G$ be an epimorphism where $G$ is finite and non-abelian. Then $\operatorname{ker}(\phi)$ is a Bieberbach group whose holonomy group has strictly smaller order than the holonomy group of $S$.

Proof. Let $1 \rightarrow \mathbb{Z}^{n-1} \rightarrow S \rightarrow F \rightarrow 1$ be such that $F$ is the holonomy group of $S$. Then $\phi\left(\mathbb{Z}^{n-1}\right)$ is normal in $G$. Consider the short exact sequence of chain complexes,

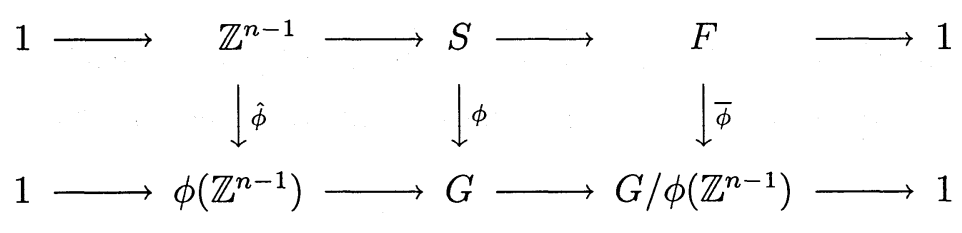

The corresponding exact sequence in homology is

$$
1 \rightarrow \operatorname{ker}(\hat{\phi}) \rightarrow \operatorname{ker}(\phi) \rightarrow \operatorname{ker}(\bar{\phi}) \rightarrow 1 \rightarrow 1 \rightarrow \operatorname{coker}(\bar{\phi}) \rightarrow 1
$$

Consequently $\bar{\phi}$ is an epimorphism and we get the following short exact sequence:

$$
1 \rightarrow \operatorname{ker}(\hat{\phi}) \rightarrow \operatorname{ker}(\phi) \rightarrow \operatorname{ker}(\bar{\phi}) \rightarrow 1
$$

Since $\operatorname{ker}(\hat{\phi})$ is free abelian and $\operatorname{ker}(\bar{\phi})$ is finite, the holonomy group of $\operatorname{ker}(\phi)$ is a quotient group of $\operatorname{ker}(\bar{\phi})$. But $\operatorname{order}(\operatorname{ker} \bar{\phi})<\operatorname{order}(F)$ since the quotient group $F / \operatorname{ker}(\bar{\phi}) \simeq G / \phi\left(\mathbb{Z}^{n-1}\right)$ is non-trivial because $G$ is non-abelian and $\phi\left(\mathbb{Z}^{n-1}\right)$ is abelian.

Corollary 2.4. Let $X^{n}$ be a complete, non-compact, finite volume real hyperbolic manifold. Then there exists a finite sheeted covering space of $X$ whose cusps are all diffeomorphic to $T^{n-1} \times[0, \infty)$ where $T^{n-1}$ is the $(n-1)$-torus.

Proof. Let $C_{i}$ be a cusp of $X$ whose cross-section $T_{i}$ is not an $(n-1)$-torus. Since the number of cusps is finite, $i=1,2, \ldots, k$ enumerates all such cusps. Then, by the structure theorem for Bieberbach groups, we can choose two elements $a_{i}, b_{i}$ in $\pi_{1}\left(C_{i}\right)$ which do not commute. Let $g_{i}=\left[a_{i}, b_{i}\right](\neq 1)$. By residual finiteness of $\pi_{1}(X)$ we can find a finite group $G_{i}$ and an epimorphism $\phi_{i}: \pi_{1}(X) \rightarrow G_{i}$ such that $\phi_{i}\left(g_{i}\right) \neq 1$. Clearly $G_{i}$ is not abelian. In fact, $\phi_{i}\left(\pi_{1}\left(C_{i}\right)\right)$ is not abelian. Let $G=G_{1} \times G_{2} \times \cdots \times G_{k}$ and $\phi: \pi_{1}(X) \rightarrow G$ be 
$\phi_{1} \times \phi_{2} \times \cdots \times \phi_{k}$. Now, for the finite sheeted covering of $X$ corresponding to $\operatorname{ker}(\phi)$, by Lemma 2.3, the fundamental groups of the cusps lying over $C_{i}$ have smaller order holonomy than that of $\pi_{1}\left(C_{i}\right)$ since $\phi\left(\pi_{1}\left(C_{i}\right)\right)$ is non-abelian. Hence the maximum order holonomy of the fundamental group of a cusp of this cover is smaller than the maximum order holonomy of the fundamental group of a cusp of $X$. Therefore, repeating this procedure, after a finite number of times we get a finite covering of $X$ in which the fundamental groups of the cusps are all equal $\mathbb{Z}^{n-1}$.

Lemma 2.5. (Borel $[\mathrm{B}]$ ) In each dimension $n \geq 2$, there exists a complete, real hyperbolic $n$-dimensional manifold which has finite volume but is not compact.

Lemma 2.6. (cf. [FJ4, Lemma 4]) Let $T^{n},(n>4)$ be a closed flat manifold which is also stably parallelizable. Let $\Sigma^{n}$ be a homotopy sphere which is not diffeomorphic to $S^{n}$. Then the connected sum $T^{n} \# \Sigma^{n}$ is not diffeomorphic to any closed flat manifold.

Proof of Theorem 2.1. Since the cross-section of the distinguished neck of $M$ is an $(m-1)$-torus $T^{m-1}$, in the new manifold $M_{f, \phi}$, this cross section becomes $T^{m-1} \# \Sigma^{m-1}$. Note that $\mathbb{Z}^{m-1} \subset \pi_{1}(M) \simeq \pi_{1}\left(M_{f, \phi}\right)$. Suppose, now, that $M_{f, \phi}$ admits a Riemannian metric of nonpositive sectional curvature. Then the Flat torus theorem [EHS, §6] implies that the covering space $\mathcal{C}$ of $M_{f, \phi}$ corresponding to the subgroup $\mathbb{Z}^{m-1}$ of $\pi_{1}\left(M_{f, \phi}\right)$ is diffeomorphic to $T^{m-1} \times \mathbb{R}$. But, being the covering space over $M_{f, \phi}, \mathcal{C}$ also has $T^{m-1} \# \Sigma^{m-1}$ embedded in it as a deformation retract. Since both $T^{m-1}$ and $T^{m-1} \# \Sigma^{m-1}$ are compact there is a smooth $h$-cobordism between them in $\mathcal{C}$. But then they must be diffeomorphic because the Whitehead group $W h \mathbb{Z}^{m-1}=0$ which contradicts Lemma 2.6. Hence the theorem.

Remark 2.7. Lemmas 2.5,2.2 and Corollary 2.4 show that in each dimension $m \geq 7$ there is an $m$-dimensional manifold $M$ satisfying the hypotheses of Theorem 2.1. 


\section{EXAMPLES FROM SCHROEDER'S CONSTRUCTION}

Schroeder's construction [S], which involves modification of a finite volume real hyperbolic manifold near the cusp, is a variation of Thurston's cusp closing result for hyperbolic 3-manifolds which generalises to higher dimensions also. Consider an $n$-dimensional ( $n \geq 4$ ) real hyperbolic manifold $M$ of finite volume and one cusp $C$ diffeomorphic to $T^{n-1} \times[0, \infty)$ where $T^{n-1}$ is an $(n-1)$ torus. Now cut off the cusp to obtain a manifold $\bar{M}$ with torus $T^{n-1}$ as boundary. This boundary is then closed by a tube $\mathbb{D}^{2} \times T^{n-2}$ around a flat torus of codimension 2 by identifying $\partial\left(\mathbb{D}^{2} \times T^{n-2}\right)=S^{1} \times T^{n-2}$ with $T^{n-1}$ by a diffeomorphism of $T^{n-1}$. (Caveat: The notation $\mathbb{D}^{2} \times T^{n-2}$ and $S^{1} \times$ $T^{n-2}$ denotes the product smooth manifolds but not product metrics.) We then say that the cusp $C$ can be closed relative to $\alpha$ where $\alpha \in \pi_{1}\left(T^{n-1}\right)=$ $\pi_{1}(C)$ is a basis element and is topologically represented by $S^{1} \times *$ in the above identification. After glueing and smoothing the metric we get a compact smooth Riemannian manifold $\hat{M}(\alpha)$ with sectional curvature $K \leq 0$ which contains a totally geodesic embedded flat torus $T^{n-2} \subset \hat{M}(\alpha)$ of codimension 2 such that the sectional curvature is strictly negative on all 2-planes which are not tangent to $T^{n-2}$. The closing of the cusp is possible because of the following lemma which is implicit in Schroeder's paper.

Lemma 3.1. (Schroeder [S]) Given $n$, there exists an $l>0$ such that for any finite volume, complete, real hyperbolic manifold $M$ of dimension $n$ and any cusp $C$ on $M$ with torus cross-section, the cusp $C$ can be closed relative to $\alpha \in \pi_{1}\left(T^{n-1}\right)$ where $T^{n-1}$ is a cross-section of $C$, provided that the length of a closed geodesic in the homotopy class of $\alpha$ in $T^{n-1}$ is bigger than or equal to $l$.

We allow, more generally, for the possibilty that the real hyperbolic hyperbolic manifold $M$ has more than one cusp (cf. Corollary 2.4). So, we modify the above notation to $M$ having finitely many cusps $C_{i}=T_{i}^{n-1} \times[0, \infty), \bar{M}=$ $M-\bigcup_{i=1}^{k} T_{i}^{n-1} \times\left[a_{i}, \infty\right)$ for $a_{i} \in[0, \infty)$ and $\hat{M}$ is the resulting manifold obtained after applying Schroeder's construction to each cusp $C_{i}$, i.e., closing them by $\mathbb{D}^{2} \times T_{i}^{n-2}$ where $T_{i}^{n-2}$ for $i=1,2, \ldots, k$, is a flat torus of codimension 2. We also identify $T_{i}^{n-1}$ with $T_{i}^{n-1} \times a_{i}$ and assume that $M$ is orientable. 
Lemma 3.1 necessitates the following lemma to make room for the closing of a cusp.

Lemma 3.2. With the notation as above, by going to a finite sheeted cover of $M$ we can assume that the torus $T_{i}^{n-1} \times a_{i}$ in the cusp $C_{i}$, for all $i$, of $M$ has injectivity radius $\geq l / 2$; l as in Lemma 3.1 .

Proof. Let $\gamma_{1}^{i}, \ldots ., \gamma_{r}^{i}$ be a list (up to free homotopy) of all closed geodesics in $T_{i}^{n-1} \times a_{i}$ which have length less than or equal to $l$. Choose a point $q \in M$ and elements $\alpha_{1}^{i}, \ldots, \alpha_{r}^{i}$ in $\pi_{1}(M, q)$ such that $\alpha_{j}^{i}$ is freely homotopic to $\gamma_{j}^{i}$ $(j=1, \ldots, r)$. Since $\pi_{1}(M, q)$ is residually finite, there is a homomorphism $h_{i}: \pi_{1}(M, q) \rightarrow G_{i}$ onto a finite group $G_{i}$ such that $h_{i}\left(\alpha_{j}^{i}\right) \neq 1$ for all $i, j$. Let $G=G_{1} \times \cdots \times G_{k}$ and $h=h_{1} \times \cdots \times h_{k}$ and consider the map $h: \pi_{1}(M, q) \rightarrow G$. Now, the finite sheeted covering space of $M$ corresponding to the kernel of $h$ has the following property. If $C$ is a cusp of this cover lying over $C_{i}(i=1, \ldots ., k)$ then the cross section of $C$ lying over $T_{i}^{n-1} \times a_{i}$ has injectivity radius $\geq l / 2$.

We now prove the following Homology lemma which will be used later in changing the smooth structure on $\hat{M}$.

Lemma 3.3. For each prime $p$, there exist elements $x_{i} \in H_{1}\left(T_{i}^{n-1}, \mathbb{Z}\right)$ such that

1. $\sum_{i=1}^{k} \overline{x_{i}}=0$ where $\overline{x_{i}}$ is the image of $x_{i}$ in $H_{1}\left(\bar{M}, \mathbb{Z}_{p}\right)$.

2. $\bigoplus_{i=1}^{k} x_{i} \in \bigoplus_{i=1}^{k} H_{1}\left(T_{i}^{n-1}, \mathbb{Z}\right)$ is a basis element.

Proof. We first observe that to prove the lemma it is enough to show that there exist $y_{i} \in H_{1}\left(T_{i}^{n-1}, \mathbb{Z}\right)$ such that $\sum_{i=1}^{k} \overline{y_{i}}=0$ and $\overline{y_{i}} \neq 0$ for some $i$. To verify this let $\bigoplus_{i=1}^{k} y_{i}=d\left(\bigoplus_{i=1}^{k} x_{i}\right)$ where $\bigoplus_{i=1}^{k} x_{i}$ is a basis element and $d(\neq 0) \in \mathbb{Z}$. If $p \mid d$, then $\bigoplus_{i=1}^{k} \overline{y_{i}}=d\left(\bigoplus_{i=1}^{k} \overline{x_{i}}\right)=0$ and hence $\overline{y_{i}}=0$ for all $i=1,2, \ldots, k$ which contradicts the assumption that $\overline{y_{i}} \neq 0$ for some $i$. Therefore, $(p, d)=1$. We then have, $0=\Sigma_{i=1}^{k} \overline{y_{i}}=\bar{d} \Sigma_{i=1}^{k} \overline{x_{i}}$ where $\bar{d}=d+p \mathbb{Z}$ which is an unit in $\mathbb{Z}_{p}$. This implies $\Sigma_{i=1}^{k} \overline{x_{i}}=0$ thereby proving the lemma.

Now, since $H_{1}\left(T_{i}^{n-1}, \mathbb{Z}\right) \rightarrow H_{1}\left(T_{i}^{n-1}, \mathbb{Z}_{p}\right)$ is an epimorphism, we only need to find elements $z_{i} \in H_{1}\left(T_{i}^{n-1}, \mathbb{Z}_{p}\right)$ such that some $z_{i} \neq 0$ and $\sum_{i=1}^{k} \hat{z}_{i}=$ 0 where $\hat{z}_{i}$ is the image of $z_{i}$ in $H_{1}\left(\bar{M}, \mathbb{Z}_{p}\right)$. Suppose on the contrary that there does not exist any such $z_{i}$ 's. This means that $\sum_{i=1}^{k} \hat{z}_{i}=0$ implies each 
$z_{i}=0$; in other words, the map $\phi_{*}: H_{1}\left(\partial \bar{M}, \mathbb{Z}_{p}\right) \rightarrow H_{1}\left(\bar{M}, \mathbb{Z}_{p}\right)$ is monic where $\phi: \partial \bar{M} \rightarrow \bar{M}$ is the inclusion map. This is equivalent to saying that $\phi^{*}: H^{1}\left(\bar{M}, \mathbb{Z}_{p}\right) \rightarrow H^{1}\left(\partial \bar{M}, \mathbb{Z}_{p}\right)$ is an epimorphism. Let $b_{i j} \in H^{1}\left(T_{i}^{n-1}, \mathbb{Z}_{p}\right), j=$ $1, \ldots, n-1 ; i=1, \ldots, k$, be such that the cup product $b_{i 1} \cup b_{i 2} \cup \cdots \cup b_{i n-1}$ generates $H^{n-1}\left(T_{i}^{n-1}, \mathbb{Z}_{p}\right)$ for each $i=1,2, \ldots ., k$. Let $r_{i}: \partial \bar{M} \rightarrow T_{i}^{n-1}$ be the unique map (up to homotopy) such that $r_{i}$ restricted to $T_{i}^{n-1}$ is the identity map and $r_{i}$ restricted to $T_{j}^{n-1}$ is a constant map for all $j \neq i$. Let $\overline{b_{i j}}=$ $r_{i}^{*}\left(b_{i j}\right)$ where $r_{i}^{*}: H^{*}\left(T_{i}^{n-1}, \mathbb{Z}_{p}\right) \rightarrow H^{*}\left(\partial \bar{M}, \mathbb{Z}_{p}\right)$ is the induced cohomology map. Further, since $\phi^{*}$ is an epimorphism, let $B_{i j} \in H^{1}\left(\bar{M}, \mathbb{Z}_{p}\right)$ be such that $\phi^{*}\left(B_{i j}\right)=\overline{b_{i j}}$. Put $B_{i 1} \cup \cdots \cup B_{i n-1}=B_{i} ; \overline{b_{i 1}} \cup \cdots \cup \overline{b_{i n-1}}=\overline{b_{i}}$ and $b_{i 1} \cup \cdots \cup b_{i n-1}=b_{i}$. Therefore, $\phi^{*}\left(B_{i}\right)=\overline{b_{i}}$ and $r_{i}^{*}\left(b_{i}\right)=\overline{b_{i}}$.

Claim: $\overline{b_{1}}, \overline{b_{2}}, \ldots ., \overline{b_{k}}$ generates $H^{n-1}\left(\partial \bar{M}, \mathbb{Z}_{p}\right)$.

We defer the proof of the claim for the moment and finish the proof of the lemma using the claim. Clearly, it follows from the claim that the map $\phi^{*}: H^{n-1}\left(\bar{M}, \mathbb{Z}_{p}\right) \rightarrow H^{n-1}\left(\partial \bar{M}, \mathbb{Z}_{p}\right)$ is an epimorphism. Now look at the cohomology exact sequence, $H^{n-1}\left(\bar{M}, \mathbb{Z}_{p}\right) \rightarrow H^{n-1}\left(\partial \bar{M}, \mathbb{Z}_{p}\right) \rightarrow H^{n}\left(\bar{M}, \partial \bar{M}, \mathbb{Z}_{p}\right) \rightarrow$ $H^{n}\left(\bar{M}, \mathbb{Z}_{p}\right) \rightarrow H^{n}\left(\partial \bar{M}, \mathbb{Z}_{p}\right)$. Since $H^{n}\left(\partial \bar{M}, \mathbb{Z}_{p}\right)=0$, it follows that

$$
H^{n}\left(\bar{M}, \partial \bar{M}, \mathbb{Z}_{p}\right) \simeq H^{n}\left(\bar{M}, \mathbb{Z}_{p}\right) .
$$

Also, by using Lefschetz duality, we have

$$
H^{n}\left(\bar{M}, \partial \bar{M}, \mathbb{Z}_{p}\right) \simeq H_{0}\left(\bar{M}, \mathbb{Z}_{p}\right) \text { and } H^{n}\left(\bar{M}, \mathbb{Z}_{p}\right) \simeq H_{0}\left(\bar{M}, \partial \bar{M}, \mathbb{Z}_{p}\right) .
$$

But then, $H_{0}\left(\bar{M}, \mathbb{Z}_{p}\right)=\mathbb{Z}_{p}$ and since $H_{0}\left(\partial \bar{M}, \mathbb{Z}_{p}\right) \rightarrow H_{0}\left(\bar{M}, \mathbb{Z}_{p}\right)$ is onto, $H_{0}\left(\bar{M}, \partial \bar{M}, \mathbb{Z}_{p}\right)=0$ which is impossible. This concludes the proof of the lemma.

Proof of the Claim: Since $H^{n-1}\left(\partial \bar{M}, \mathbb{Z}_{p}\right)=\bigoplus_{i=1}^{k} H^{n-1}\left(T_{i}^{n-1}, \mathbb{Z}_{p}\right)$ and $k=$ $\operatorname{dim}_{\mathbb{Z}_{p}} H^{n-1}\left(\partial \bar{M}, \mathbb{Z}_{p}\right)=$ number of components of $\partial \bar{M}$, we need only show that $\overline{b_{i}}, i=1, \ldots, k$, are linearly independent. Let $\sigma_{i}: T_{i}^{n-1} \rightarrow \partial \bar{M}$ be the inclusion map. Note that $r_{i} \circ \sigma_{j}=$ identity on $T_{i}^{n-1}$ if $i=j$ and is a constant map if $i \neq j$. Suppose $\sum_{i=1}^{k} s_{i} \overline{b_{i}}=0$ where $s_{i} \in \mathbb{Z}_{p}$. Applying $\sigma_{j}^{*}$, we have

$$
\sigma_{j}^{*}\left(\sum_{i=1}^{k} s_{i} \overline{b_{i}}\right)=\sum_{i=1}^{k} s_{i} \sigma_{j}^{*}\left(r_{i}^{*}\left(b_{i}\right)\right)=\sum_{i=1}^{k} s_{i}\left(r_{i} \circ \sigma_{j}\right)^{*}\left(b_{i}\right)=0 .
$$


Therefore, $s_{j} b_{j}=0$, which is an equation in $H^{n-1}\left(T_{j}^{n-1}, \mathbb{Z}_{p}\right)=\mathbb{Z}_{p}$ for which $b_{j}$ is a generator. This implies $s_{j}=0$. Thus $\left\{\bar{b}_{i} ; i=1, \ldots ., k\right\}$ are linearly independent which concludes the proof of the claim.

Pick a prime $p \neq 2$ and such that $p \mid \operatorname{order}\left(\Theta_{n-2}\right)$ (e.g., $n=9$ and $\left.p=7\right)$. We assume through the remainder of this paper that $n \geq 9$. Let $x_{i} \in H_{1}\left(T_{i}^{n-1}, \mathbb{Z}\right)$ be as in Lemma 3.3. Write $x_{i}=d_{i} y_{i}$ where $y_{i}$ is a basis element in $H_{1}\left(T_{i}^{n-1}, \mathbb{Z}\right)$ and $d_{i} \geq 1$ is an integer. Put $\hat{M}\left(y_{1}, \ldots ., y_{k}\right)=\hat{M}$ where $\hat{M}\left(y_{1}, \ldots ., y_{k}\right)$ is the compact manifold obtained after applying Schroeder's construction to $\bar{M}$ by closing the cusps $C_{i}$ relative to basis elements $y_{i}$. (See Lemma 3.2.) We then have,

Theorem 3.4. There exists a smooth manifold $\mathcal{M}$ such that

1. $\mathcal{M}$ is homeomorphic to $\hat{M}=\hat{M}\left(y_{1}, \ldots ., y_{k}\right)$.

2. $\mathcal{M}$ does not support any nonpositively curved metric.

Proof. Let $\Sigma \in \Theta_{n-2}=\left[S^{n-2}, T O P / 0\right]$ such that $\operatorname{order} \Sigma=p$. To produce $\mathcal{M}$ we find a map $\mathcal{M} \in[\hat{M}, T O P / 0]$. Note that $\hat{M}=\bar{M} \cup_{i=1}^{k} T_{i}^{n-2} \times \mathbb{D}^{2}$ where $T_{i}^{n-1}$ is identified with $\partial\left(T_{i}^{n-2} \times \mathbb{D}^{2}\right)$ so that $* \times S^{1}$ represents $y_{i} \in H_{1}\left(T_{i}^{n-1}, \mathbb{Z}_{p}\right)$.

We will show that there exists a continuous map $\mathcal{M}: \hat{M} \rightarrow T O P / 0$ such that $\mathcal{M}$ restricted to $T_{i}^{n-2} \times \mathbb{D}^{2}$ is a composite of the maps

$$
T_{i}^{n-2} \times \mathbb{D}^{2} \stackrel{P_{i}}{\rightarrow} T_{i}^{n-2} \stackrel{\phi_{i}}{\rightarrow} S^{n-2} \stackrel{d_{i}}{\rightarrow} S^{n-2} \stackrel{\Sigma}{\rightarrow} T O P / 0
$$

where $P_{i}$ is the projection map, $\phi_{i}$ is the degree 1 map, $d_{i}$ is the degree $d_{i}$ map and $\Sigma$ is the exotic structure on $S^{n-2}$ regarded as an element in $\left[S^{n-2}, T O P / 0\right]$. (Note that the homotopy classes of $\phi_{i}, d_{i}$, and $\Sigma$ are uniquely defined.)

Recall that $S^{n-2}$ is contained in the Eilenberg-Maclane space $K\left(n-2, \mathbb{Z}_{p}\right)$ and any element in $H^{n-2}\left(\hat{M}, \mathbb{Z}_{p}\right)$ is actually a map $\hat{M} \rightarrow K\left(n-2, \mathbb{Z}_{p}\right)$. And by Cellular approximation theorem the image of such a map resides in the $n$-skeleton of $K\left(n-2, \mathbb{Z}_{p}\right)$ which, for odd primes $p$ and $n \geq 9$, is the Moore space $S^{n-2} \cup_{p} \mathbb{D}^{n-1}$. On the other hand, since order $\Sigma=p$ in the group $\Theta_{n-2}=$ $\pi_{n-2}(T O P / 0), \Sigma$ extends to a map $\eta: S^{n-2} \cup_{p} \mathbb{D}^{n-1} \rightarrow T O P / 0$. Therefore, by composing $\eta$ with a suitable element in $H^{n-2}\left(\hat{M}, \mathbb{Z}_{p}\right)$ we get a map $\mathcal{M}: \hat{M} \rightarrow$ 
$T O P / 0$. This is illustrated in the following diagram:

$$
\begin{aligned}
& K\left(n-2, \mathbb{Z}_{p}\right) \\
& \uparrow \sigma \\
& \hat{M} \\
& \uparrow \sigma_{i} \\
& T_{i}^{n-2} \times \mathbb{D}^{2} \stackrel{P_{i}}{\rightarrow} T_{i}^{n-2} \stackrel{\phi_{i}}{\rightarrow} S^{n-2} \stackrel{d_{i}}{\rightarrow} \\
& S^{n-2} \cup_{p} \mathbb{D}^{n-1} \\
& \uparrow \tau \quad \searrow \eta \\
& S^{n-2} \stackrel{\Sigma}{\longrightarrow} T O P / 0
\end{aligned}
$$

Define $\overline{\phi_{i}}=\tau \circ d_{i} \circ \phi_{i} \circ P_{i}$ and $\hat{\phi}_{i}=\sigma \circ \overline{\phi_{i}}$ where $\sigma, \tau$ and $\sigma_{i}$ are the inclusion maps.

Claim: There exists $\hat{\phi} \in H^{n-2}\left(\hat{M}, \mathbb{Z}_{p}\right)$ such that $\left.\hat{\phi}\right|_{T_{i}^{n-2} \times \mathbb{D}^{2}}=\hat{\phi}_{i}$.

This amounts to showing that there exists a map $\hat{\phi}: \hat{M} \rightarrow K\left(n-2, \mathbb{Z}_{p}\right)$ and from our remark earlier we actually have $\hat{\phi}=\sigma \circ \bar{\phi}$ where $\bar{\phi}$ is a map $\hat{M} \rightarrow S^{n-2} \cup_{p} \mathbb{D}^{n-1}$. Further, $\eta \circ \overline{\phi_{i}}=\Sigma \circ d_{i} \circ \phi_{i} \circ P_{i}$ since $\eta \circ \tau=\Sigma$. Therefore, by defining $\mathcal{M}=\eta \circ \bar{\phi}$, we do the construction posited in the second paragraph of this proof.

Proof of the Claim: Since $\bar{M} \subset \hat{M}$, we have the following commutative cohomology diagram:

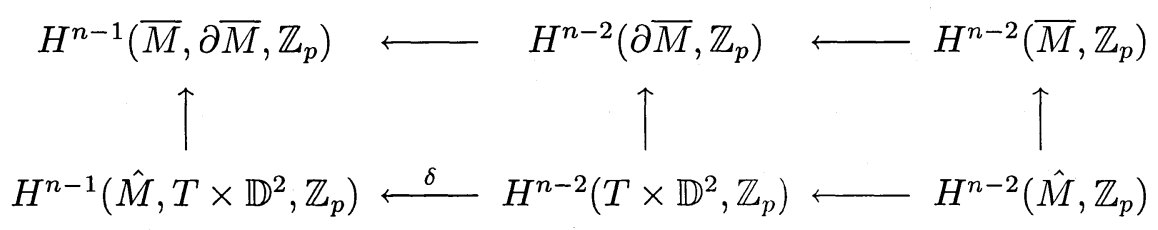

where $T=\amalg T_{i}^{n-2}$ and $H^{n-1}\left(\bar{M}, \partial \bar{M}, \mathbb{Z}_{p}\right) \simeq H^{n-1}\left(\hat{M}, T \times \mathbb{D}^{2}, \mathbb{Z}_{p}\right)$ and also note that $H^{n-2}\left(T \times \mathbb{D}^{2}, \mathbb{Z}_{p}\right)=\bigoplus_{i=1}^{k} H^{n-2}\left(T_{i}^{n-2} \times \mathbb{D}^{2}, \mathbb{Z}_{p}\right)$ and $H^{n-2}\left(\partial \bar{M}, \mathbb{Z}_{p}\right)=$ $\bigoplus_{i=1}^{k} H^{n-2}\left(T_{i}^{n-1}, \mathbb{Z}_{p}\right)$. Now $\bigoplus_{i=1}^{k} \hat{\phi}_{i} \in H^{n-2}\left(T \times \mathbb{D}^{2}, \mathbb{Z}_{p}\right)$. It is enough to show that $\delta\left(\oplus \hat{\phi}_{i}\right)=0$ which proves that there exists a $\hat{\phi} \in H^{n-2}\left(\hat{M}, \mathbb{Z}_{p}\right)$ such that $\hat{\phi} \mapsto \oplus \hat{\phi}_{i}$. This is equivalent to showing that the image of $\oplus \hat{\phi}_{i}$ in $H^{n-1}\left(\bar{M}, \partial \bar{M}, \mathbb{Z}_{p}\right)$ via $H^{n-2}\left(\partial \bar{M}, \mathbb{Z}_{p}\right)$ is zero which again, from the following commutative diagram (which we get by using Lefschetz duality)

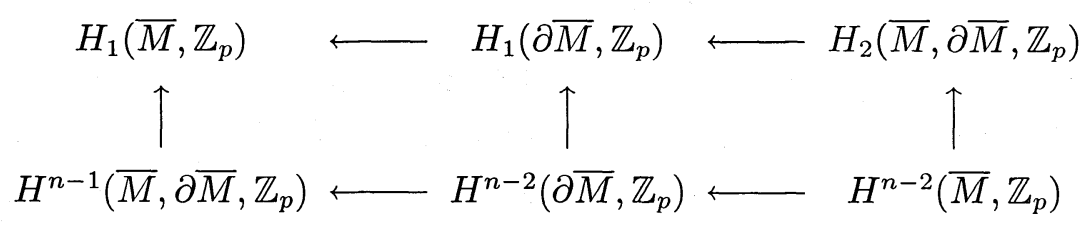


is equivalent to showing that the image of the element $\oplus \hat{\phi}_{i}$ in $H_{1}\left(\bar{M}, \mathbb{Z}_{p}\right)$ via $H^{n-2}\left(\partial \bar{M}, \mathbb{Z}_{p}\right)$ and $H_{1}\left(\partial \bar{M}, \mathbb{Z}_{p}\right)$ is zero. Let $\hat{\alpha}_{i}$ denote the image of $x_{i}$ under the homomorphism determined by the change of homology coefficients $\mathbb{Z} \rightarrow \mathbb{Z}_{p}$. Then, by our construction, the image of $\oplus \hat{\phi}_{i}$ in $H_{1}\left(\partial \bar{M}, \mathbb{Z}_{p}\right)$ is $\oplus \hat{x}_{i}$ which, by Lemma 3.3 , goes to $\Sigma \overline{x_{i}}=0$ in $H_{1}\left(\bar{M}, \mathbb{Z}_{p}\right)$. This concludes the proof of the claim.

We now proceed to show that $\mathcal{M}$ cannot support any nonpositively curved metric. Suppose on the contrary it does.

Since $T_{i}^{n-2}$ is totally geodesically embedded in $\hat{M}, \pi_{1}\left(T_{i}^{n-2}\right)=\mathbb{Z}^{n-2}$ is a subgroup of $\pi_{1}(\hat{M})$. Let $\hat{M}_{i}$ denote the covering space of $\hat{M}$ corresponding to $\pi_{1}\left(T_{i}^{n-2}\right)$. Note that $T_{i}^{n-2}$ lifts to a unique totally geodesic submanifold of $\hat{M}_{i}$ which we identify with $T_{i}^{n-2}$ and by [BO, Lemma 3.1] the normal bundle $\nu T_{i}^{n-2}$ of $T_{i}^{n-2}$ is diffeomorphic, via the exponential map, to $\hat{M}_{i}$. But the normal bundle of $T_{i}^{n-2}$ in $\hat{M}_{i}$ is the same as the normal bundle of $T_{i}^{n-2}$ in $\hat{M}$. Therefore, $\nu T_{i}^{n-2}$ is diffeomorphic to $T_{i}^{n-2} \times \mathbb{R}^{2}$ which means that $\hat{M}_{i}$ is diffeomorphic to $T_{i}^{n-2} \times \mathbb{R}^{2}$. Now $\mathcal{M}$ is another smooth structure on $\hat{M}$ given by $\mathcal{M}: \hat{M} \rightarrow T O P / 0$ such that $\left.\mathcal{M}\right|_{T_{i}^{n-2} \times \mathbb{D}^{2}}=\Sigma \circ d_{i} \circ \phi_{i} \circ P_{i}$. Hence $\left.\mathcal{M}\right|_{T_{i}^{n-2} \times \text { Int } \mathbb{D}^{2}}$ is diffeomorphic to $\left(T_{i}^{n-2} \# d_{i} \Sigma\right) \times \mathbb{R}^{2}$.

Let $\mathcal{M}_{i}$ be the cover of $\mathcal{M}$ corresponding to $\pi_{1}\left(T_{i}^{n-2}\right) \subset \pi_{1}(\hat{M})=\pi_{1}(\mathcal{M})$. Then this smooth structure on $\hat{M}_{i}$ corresponds to the map $\mathcal{M} \circ p_{i}: \hat{M}_{i} \rightarrow \hat{M} \rightarrow$ $T O P / 0$ where $p_{i}$ is the covering map. Also, $T_{i}^{n-2} \times \mathbb{D}^{2} \subset \hat{M}_{i}$ is a homotopy equivalence. From this we conclude that

$$
\mathcal{M}_{i} \text { is diffeomorphic to }\left(T_{i}^{n-2} \# d_{i} \Sigma\right) \times \mathbb{R}^{2} .
$$

Since $\mathcal{M}$ is nonpositively curved, by our hypothesis, and closed we can apply Flat torus theorem to $\pi_{1}\left(T_{i}^{n-2}\right) \subset \pi_{1}(\hat{M})=\pi_{1}(\mathcal{M})$ to get an embedded totally geodesic flat torus $\mathcal{T}_{i} \subset \mathcal{M}_{i}$ inducing a homotopy equivalence. Therefore, by using [BO, Lemma 3.1] once again, we see that

$$
\mathcal{M}_{i} \text { is diffeomorphic to } \nu\left(\mathcal{T}_{i}\right)
$$

where $\nu\left(\mathcal{T}_{i}\right)$ is the normal bundle of $\mathcal{T}_{i}$. Because of (3.2), $\mathcal{M}_{i}$ can be smoothly compactified with $\partial=S \nu\left(\mathcal{T}_{i}\right)$, the sphere bundle of $\nu\left(\mathcal{T}_{i}\right)$. But by (3.1), $\mathcal{M}_{i}$ has a second smooth compactification with $\partial^{\prime}=\left(T_{i}^{n-2} \# d_{i} \Sigma\right) \times S^{1}$. But the 
boundaries of any two compactifications of the same space are $h$ - cobordant and since $\pi_{1}\left(\partial^{\prime}\right)$ is free abelian, the $h$-cobordism is a product; in particular, $\partial$ is diffeomorphic to $\partial^{\prime}$, i.e.,

$$
S \nu\left(\mathcal{T}_{i}\right) \text { is diffeomorphic to }\left(T_{i}^{n-2} \# d_{i} \Sigma\right) \times S^{1}
$$

Now consider the following homotopy sequence of the $S^{1}$ fiber bundle $F$ : $S \nu\left(\mathcal{T}_{i}\right) \rightarrow \mathcal{T}_{i}$

$$
\pi_{2}\left(\mathcal{T}_{i}\right) \rightarrow \pi_{1} S^{1} \rightarrow \pi_{1}\left(S \nu\left(\mathcal{T}_{i}\right)\right) \rightarrow \pi_{1} \mathcal{T}_{i} \rightarrow \pi_{0} S^{1}
$$

Clearly $\pi_{0} S^{1}$ and $\pi_{2}\left(\mathcal{T}_{i}\right)$ are both zero and by $(3.3), \pi_{1}\left(S \nu\left(\mathcal{T}_{i}\right)\right)$ is an abelian group. Also, since $\pi_{1} \mathcal{T}_{i}$ is free abelian, the map $F_{\#}: \pi_{1}\left(S \nu\left(\mathcal{T}_{i}\right)\right) \rightarrow \pi_{1} \mathcal{T}_{i}$ splits. Since $S \nu\left(\mathcal{T}_{i}\right)$ is a $K(\pi, 1)$ space, the higher homotopy vanishes by considering the rest of the homotopy sequence (3.4), this splitting is induced by a cross section to $F$. Hence the Euler class of the oriented vector bundle $\nu\left(\mathcal{T}_{i}\right)$ is 0 . Consequently, $\nu\left(\mathcal{T}_{i}\right)$ is the trivial 2 plane bundle; in particular,

$$
S \nu\left(\mathcal{T}_{i}\right)=\mathcal{T}_{i} \times S^{1}
$$

Combining (3.5) with (3.3) implies

$$
\mathcal{T}_{i} \times S^{1} \text { is diffeomorphic to }\left(T_{i}^{n-2} \# d_{i} \Sigma\right) \times S^{1} .
$$

Under this identification, since every element in $G L_{n-1}(\mathbb{Z})=\operatorname{Aut}\left(\pi_{1}\left(\mathcal{T}_{i} \times S^{1}\right)\right)$ is induced by a self-diffeomorphism of $\mathcal{T}_{i} \times S^{1}$, we can assume that $\pi_{1}\left(\mathcal{T}_{i}\right)$ is identified with $\pi_{1}\left(T_{i}^{n-2} \# d_{i} \Sigma\right)$. Take the covering space $X$ corresponding to this subgroup. Then (3.6) yields $X=\mathcal{T}_{i} \times \mathbb{R}$ and $X=\left(T_{i}^{n-2} \# d_{i} \Sigma\right) \times \mathbb{R}$, i.e.,

$$
\mathcal{T}_{i} \times \mathbb{R}=\left(T_{i}^{n-2} \# d_{i} \Sigma\right) \times \mathbb{R}
$$

Using (3.7), we obtain a smooth $h$-cobordism between $\mathcal{T}_{i}$ and $T_{i}^{n-2} \# d_{i} \Sigma$. Again since $\pi_{1} \mathcal{T}_{i}$ is free abelian, this $h$-cobordism is a product. In particular, $\mathcal{T}_{i}$ is diffeomorphic to $T_{i}^{n-2} \# d_{i} \Sigma$. But since $\mathcal{T}_{i}$ and $T_{i}^{n-2}$ are diffeomorphic we have $T_{i}^{n-2} \# d_{i} \Sigma=T_{i}^{n-2}$. This implies that $d_{i} \Sigma=0$ in $\Theta_{n-2}$. Since $\left(d_{1}, \ldots, d_{k}\right)=1$, there exist integers $a_{1}, \ldots, a_{k}$ satisfying $a_{1} d_{1}+\cdots+a_{k} d_{k}=1$. Therefore $a_{1}\left(d_{1} \Sigma\right)+\cdots+a_{k}\left(d_{k} \Sigma\right)=\Sigma$ in $\Theta_{n-2}$ and hence $\Sigma=0$ in $\Theta_{n-2}$ contradicting the assumption that it is non-zero. Therefore our assumption that 
$\mathcal{M}$ admits a metric of nonpositive curvature must be false which completes the proof of the theorem.

\section{REFERENCES}

[BBE] Ballmann, W., Brin, M. and Eberlein, P., Structure of manifolds of nonpositive curvature. I, Ann. of Math. 122 (1985), 171-203.

[BE] Ballmann, W. and Eberlein, P., Fundamental groups of manifolds of nonpositive curvature, J. Differential Geom. 25 (1987), 1-22.

[BO] Bishop, R. L. and O'Neill, B., Manifolds of negative curvature, Trans. Amer. Math. Soc. 145 (1969), 1-49.

[B] Borel, A., Compact Clifford-Klein forms of symmetric spaces, Topology 2 (1963), 111-122.

[BK] Burns, K. and Katok, A., Manifolds with nonpositive curvature, Ergodic Theory and Dynamical Systems 5 (1985), 307-317.

[EHS] Eberlein, P., Hamenstadt, U. and Schroeder, V., Manifolds of nonpositive curvature, Proc. Symposia Pure Math. 54 Part 3, 1993.

[FJ1] Farrell, F. T. and Jones, L. E., A topological analogue of Mostow's rigidity theorem, Journal of the American Math. Soc. 2 (1989), 257-370.

[FJ2] Farrell, F. T. and Jones, L. E., Negatively curved manifolds with exotic smooth structures, Journal of the American Math. Soc. 2 (1989), 899-908.

[FJ3] Farrell, F. T. and Jones, L. E., Non-uniform hyperbolic lattices and exotic smooth structures, J. Differential Geom., 38 (1993), 235-261.

[FJ4] Farrell, F. T. and Jones, L. E., Exotic smoothings of hyperbolic manifolds which do not support pinched negative curvature, Proc. Amer. Math. Soc.,to appear.

[Ma] W. Magnus, Residually finite groups, Bull. Amer. Math. Soc. 75 (1969) 305316.

[Mi] Milnor, J., Morse Theory, Annals of Math. Studies No. 51, Princeton University Press, Princeton, 1963.

[S] Schroeder, V., A cusp closing theorem, Proc. Amer. math. Soc. 106 (1989), 797-802.

Tata Institute of Fundamental Research, Bombay, India

State University of New York, Binghamton, U. S. A.

RECEIVED JUNE 8, 1993 